\title{
Reasons for Diagnostic Delay of Foot Drop Caused by Parasagittal Meningioma: Two Case Reports
}

\author{
Duc Thuan Nguyen ${ }^{a}$ Quang An Nguyen ${ }^{b}$ Thi Dung Hoang ${ }^{a}$ \\ Thanh Chung Dang ${ }^{c}$ Trung Duc Le ${ }^{a}$ \\ aDepartment of Neurology, Military Hospital 103, Vietnam Military Medical University, \\ Hanoi, Vietnam; 'bStroke Center, Phu Tho General Hospital, Phu Tho Province, Vietnam; \\ 'Department of Pathophysiology, Vietnam Military Medical University, Hanoi, Vietnam
}

\author{
Keywords \\ Foot drop $\cdot$ Parasagittal meningioma $\cdot$ Neurological examination $\cdot F$ wave $\cdot$ Recruitment
}

\begin{abstract}
Foot drop is defined as an impaired ability or inability of dorsiflexion. Peripheral nervous system injuries are commonly considered as the cause of this condition. The central causes including parasagittal meningioma are also described in the literature but very rarely and commonly not recognized early. In this article, we report 2 patients with isolated unilateral foot drop as the first symptom of a parasagittal meningioma and discuss several reasons for delayed diagnosis. Two patients were treated with decompressive craniotomy. The histopathological findings demonstrated a fibroblastic meningioma and a meningothelial meningioma. During postoperative follow-up, the woman patient showed nearly complete recovery and the second case regained total muscle power over a period of 12 months. The rarity of the disease, the absence of upper motor neuron signs, the occurrence of peripheral pathologies and misinterpretation of $\mathrm{F}$ wave on nerve conduction study, and motor unit recruitment on electromyography lead to delay in diagnosis and treatment of the central foot drop due to parasagittal meningioma.
\end{abstract}


Nguyen et al.: Central Cause of Foot Drop

\section{Introduction}

Foot drop is a condition characterized by impairment or complete loss of dorsiflexion mainly involved in the tibialis anterior, extensor digitorum longus, and extensor hallucis longus muscles innervated by both upper and lower motor neurons [1]. However, peripheral neuropathies are considered as the most frequent etiologies of foot drop and early detected. In contrast, central lesions of foot drop are rare and not promptly recognized or even misdiagnosed for various reasons [2-5]. In this article, we present 2 cases suffering from parasagittal meningioma presenting with unilateral isolated foot drop as the first symptom and discuss the reasons for delayed diagnosis.

\section{Case Presentation}

The first patient, a 37-year-old female with normal past medical history, presented a progressive left foot drop for an 8-week duration. At the initial presentation in another outpatient clinic (in August 2017), only the patient's left foot drop with grade $3 / 5$ power of ankle dorsiflexors was noted. Lumbar magnetic resonance imaging (MRI) revealed no nerve root compression. The nerve conduction study was unremarkable except for the absence of the $F$ wave in the left peroneal nerve. Electromyography (EMG) examination showed only a reduction of maximal effort recruitment in the left extensor hallucis longus and anterior tibialis. The foot drop due to peripheral origin was suspected and conservatively treated. Because of the progressively worsening foot drop, she visited our department's outpatient clinic in October 2017. Neurological examination revealed a severe left foot drop with grade $1 / 5$ strength in the foot and ankle dorsiflexion. Additionally, she had Babinski sign positive, increased muscle tone, ankle clonus, and asymmetric superficial abdominal reflex (left < right). Therefore, a cerebral MRI study was indicated and showed a right parasagittal tumor (dimension $3 \times 2 \times 2 \mathrm{~cm}$ ). Afterward, she underwent a brain tumor craniotomy (December 2017) (Fig. 1a, b). The histopathological examination demonstrated a fibroblastic meningioma (Fig. 2b). During the 30-month follow-up, she showed nearly complete recovery and no recurrent tumor.

The second patient, a 46-year-old male, complained of a sudden onset isolated foot drop on the left side. Past medical history showed no significant diseases except for intermittent low back pain radiating to bilateral lower extremities. On the initial physical examination (February 2018) at an outpatient clinic in another hospital, the left foot drop was the sole sign. On a nerve conduction study, all parameters were normal. The needle EMG study revealed purely decreased maximal voluntary effort recruitment in the left anterior tibialis and extensor hallucis longus. Lumbar MRI showed no abnormal findings. He received a diagnosis of foot drop caused by peripheral neural lesions and physiotherapy treatment. Two months later (April 2018), he came to our outpatient clinic with a progressive sign. The neurological examination revealed a slightly increased Achilles reflex, absence of left superficial abdominal reflex, and Babinski sign on the left side which suggested a central cause of foot drop. Therefore, the patient underwent a cerebral MRI study, which suggested a right parasagittal meningioma $(4 \times 5 \times 6 \mathrm{~cm})$ (Fig. 1c, d). He was treated with brain surgery to remove the tumor. The histopathological finding determined a diagnosis of a meningothelial meningioma (Fig. 2a). During 18-month postoperative follow-up, the male patient regained total muscle power.

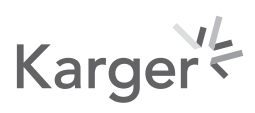


Case Reports in Neurology
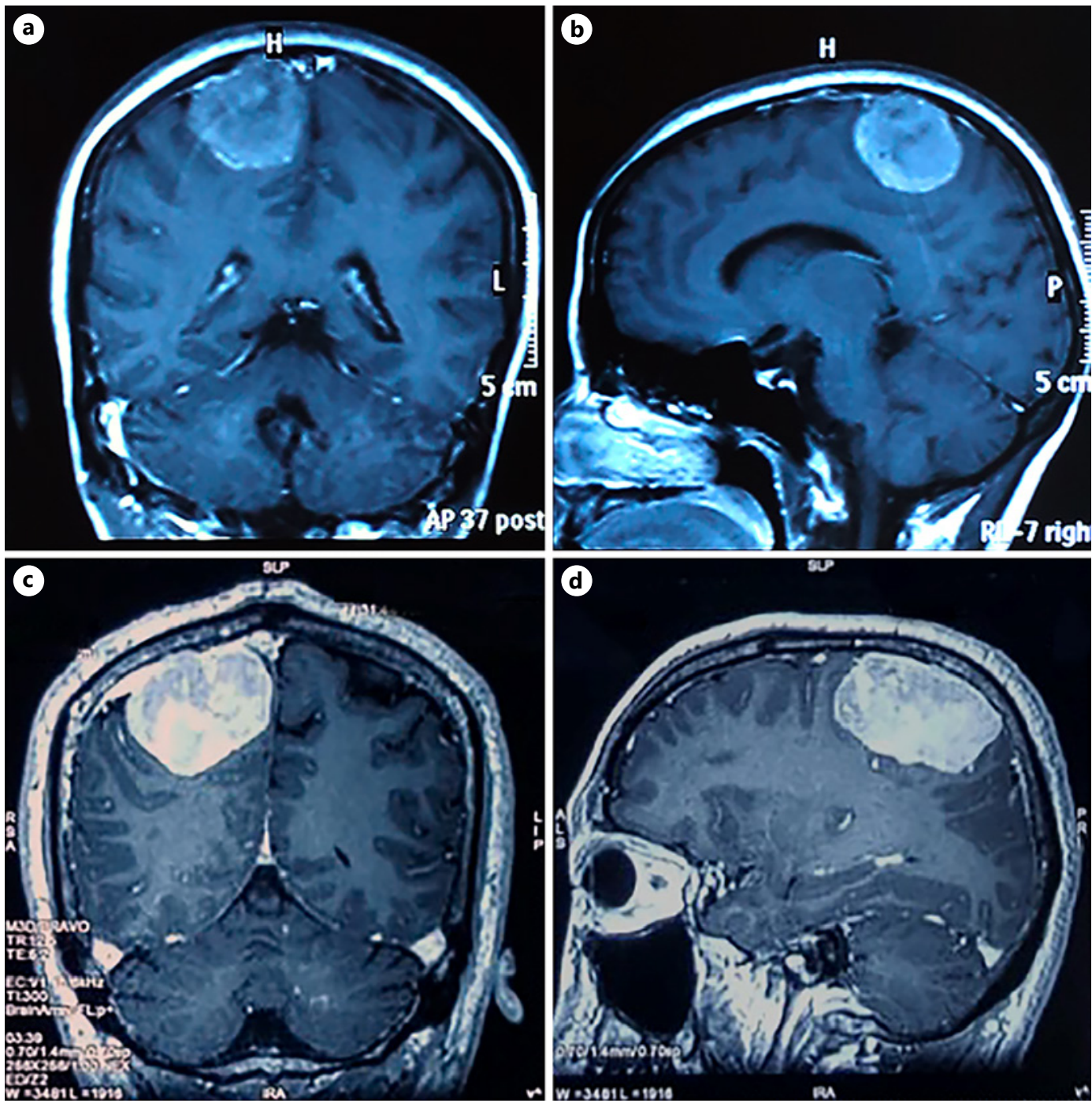

Fig. 1. Postcontrast T1-weighted axial section cerebral MRI showing a parasagittal meningioma (the first patient (a, b); the second patient $(\mathbf{c}, \mathbf{d}))$. MRI, magnetic resonance imaging.
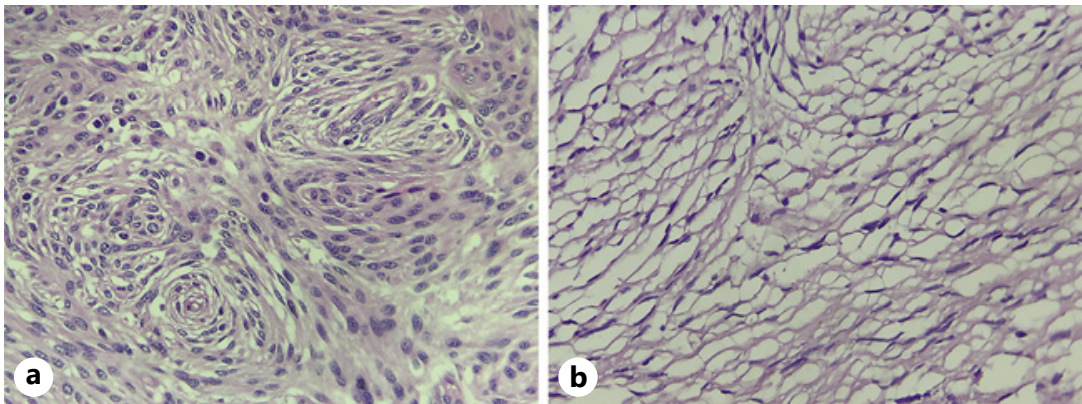

Fig. 2. Pathohistological finding showing a fibroblastic meningioma (b, the first patient) and meningothelial meningioma (a, the second patient). 
Nguyen et al.: Central Cause of Foot Drop

\section{Discussion}

Foot drop is a condition characterized by impairment or complete loss of dorsiflexion (to raise the foot or the toes from the ankle) which mainly involved 3 muscles, situated in the anterior compartment of the lower extremity, including the tibialis anterior, extensor digitorum longus, and extensor hallucis longus muscles innervated by both upper and lower motor neurons [1]. While peripheral neuropathies including anterior horn cell diseases, L5 radiculopathy, lumbosacral plexopathy, partial sciatic nerve neuropathy, and common peroneal compression neuropathy are the most frequent etiologies of foot drop and early detected, central lesions of foot drop are rare and not promptly recognized or even misdiagnosed because of a variety of reasons $[2,4,5]$. In this article, we represented 2 cases whose initial symptoms were unilateral isolated foot drop caused by central origin and discussed the reasons leading to a delay in recognizing parasagittal meningioma of the foot drop.

First, this condition is extremely rare. To the best of our knowledge, to date, there have been only 9 cases with foot drop due to a parasagittal meningioma reported in English language $[2,6,7]$, and only 2 patients with really isolated unilateral foot drop at the initial examination [4, 7]. In our country, for the first time, our 2 cases with this condition have been recorded. Although the central cause is described in classical medical textbooks, because of the scarcity of such cases, clinicians commonly do not use this knowledge in clinical practice to investigate the intracranial lesions of foot drop as in our patients [8].

Second, the reason may be due to a lack of upper motor neuron signs including hyperreflexia, decreased or absent ipsilateral superficial reflexes, spasticity, extensor plantar response (Babinski's sign), the deficit of parieto-cortical sensations, and absence of muscle atrophy [3]. At the initial examination, our patients did not show any central subjected and objected symptoms that led our colleagues to misdiagnosis as peripheral stem of foot drop. At our examination later, it became easier when Babinski's sign, exaggerated ankle jerk reflexes, increased muscle tone, clonus, and diminished abdominal superficial reflexes appeared, and parasagittal meningioma was correctly diagnosed. In addition to that, the appearance of central sensory impairment resembling peripheral causes may lead to a delay in the diagnosis of the central origin of foot drop as in a case reported by Lath and Rajshekhar [3]. Especially, if the lumbar spinal diseases were combined, the diagnosis of the central cause was often confused, even led to unnecessary operations as the cases described by Lath and colleagues [2-4].

The third reason is that the interpretation of subclinical findings is not careful enough and has not been closely combined with clinical symptoms. In our 2 cases, the comprehensive electrodiagnostic study was performed and revealed abnormality of nerve conduction (absent $\mathrm{F}$ wave in the first patient) and alternation of the EMG test (reduction of maximal effort recruitment in both cases) which was interpreted as an abnormal parameter of peripheral diseases and thus led our colleagues at initial examination not to seek the central lesion. F waves are late motor responses that result from supramaximal antidromic stimulation of lower motor neurons and can be obtained from any motor nerve. Although $\mathrm{F}$ wave responses are usually thought of as evaluating the peripheral nervous system disorders, they might be affected by central nervous system diseases such as stroke [9] and brain tumor (glioblastoma multiforme) [10]. However, until now, there have been no reports on the alternations in $\mathrm{F}$ wave parameters in patients with a parasagittal meningioma. Absent $\mathrm{F}$ responses are usually considered as a sign of proximal demyelination, but a hypoexcitability state of motoneurons could also be created through the inhibitory process of descending supraspinal pathways $[11,12]$. Thus, $\mathrm{F}$ wave absence in our patient could be a sign of the parasagittal meningioma which disrupts the focal corticospinal pathway, resulting in motoneuron hypoexcitability at the spinal level. In term of EMG results, our colleagues have found decreased maximal effort recruitment in paretic muscles but did not

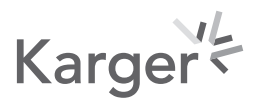


consider this finding as a presentation of central cause. In a case with a parasagittal meningioma reported by Bilić et al. [7], reduced motor unit action potential recruitment was also noted, but the authors saw it as normal result. Indeed, recruitment abnormalities were observed both in the lower motor neuron lesions and in upper motor neuron diseases. However, there are differences between the 2 disorders. It should be noted that in the central nervous system lesion, the reduction of spatial recruitment and temporal recruitment is concordant, while the combination of decreased spatial recruitment and increased temporal recruitment (faster than normally appreciable) was identified in peripheral nervous system diseases [13].

\section{Conclusion}

The scarcity of such cases, the absence of upper motor neuron signs, the occurrence of peripheral etiologies and misinterpretation of $\mathrm{F}$ wave on the nerve conduction study, and motor unit recruitment on EMG can be the main causes leading to the delayed diagnosis. To differentiate central from peripheral causes of foot drop, the anamnesis with the absence of pain and other sensory symptoms, careful interpretation of $\mathrm{F}$ wave in close association with upper motor neuron signs (e.g., Babinski sign) should be taken into attention. Moreover, quantitative EMG in the evaluation of the frequency of motor unit action potential discharge is a useful tool in this situation.

\section{Acknowledgements}

We would like to thank all staffs of the Neurology Department, Military Hospital 103 for their support to complete this study.

\section{Statement of Ethics}

Written informed consent was obtained from the patients for publication of these case reports and any accompanying images.

\section{Conflict of Interest Statement}

The authors declare that there is no conflict of interest regarding the publication of this article.

\section{Funding Sources}

The authors did not receive any funding.

\section{Authors Contributions}

N.D.T., D.T.C., and N.Q.A.: conceptualization, methodology, and writing - original draft, review, and editing; L.T.D., H.T.D.: resources, investigation, and formal analysis.

\section{Karger ${ }^{\prime}=$}




\section{References}

1 Stewart JD. Foot drop: where, why and what to do? Pract Neurol. 2008;8(3):158-69.

2 Narenthiran G, Leach P, Holland JP. Clinical features of central isolated unilateral foot drop: a case report and review of the literature. Surg Neurol Int. 2011;2(27).

3 Lath R, Rajshekhar V. Unilateral foot drop. Postgrad Med J. 1996;72(851):573-4.

4 Tun K, Türkoğlu ÖF. Foot drop as a result of bilateral parasagittal meningioma: a case report. Turk Neurosurg. 2006;16(2):94-6.

5 Westhout FD, Pare LS, Linskey ME. Central causes of foot drop: rare and underappreciated differential diagnoses. J Spinal Cord Med. 2007;30(1):62-6.

6 Bhatoe HS. Wrist drop and foot drop in cerebral cortical lesions. Med J Armed Forces India. 1998;54(2):167-9.

7 Bilić $\mathrm{H}$, Hančević M, Sitaš B, Bilić E. A rare case of parasagittal meningioma causing isolated foot drop: case report and review of the literature. Acta Neurol Belg. 2021;121(2):555-9.

8 Ozdemir N, Citak G, Acar UD. Spastic foot drop caused by a brain tumour: a case report. Br J Neurosurg. 2004; 18(3):314-5.

9 Lukács M. F wave measurements detecting changes in motor neuron excitability after ischaemic stroke. Electromyogr Clin Neurophysiol. 2007;47(2):109-15.

10 Pichiorri F, Onesti E, Tartaglia G, Inghilleri M. Foot drop of central origin: a misleading alteration of nerve conduction study. Neurol Sci. 2016;37(5):811-3.

11 Mesrati F, Vecchierini MF. F-waves: neurophysiology and clinical value. Neurophysiol Clin. 2004;34(5): 217-43.

12 Drory VE, Neufeld MY, Korczyn AD. F-wave characteristics following acute and chronic upper motor neuron lesions. Electromyogr Clin Neurophysiol. 1993;33(7):441-6.

13 Ferrante MA. The needle EMG manifestations of pathology. Comprehensive electromyography: with clinical correlations and case studies. Cambridge, UK: Cambridge University Printing House; 2018. 